Sleeter, Christine (2018). La transformación del currículo en una sociedad diversa: ¿quién y cómo se decide el currículum?. RELIEVE, 24(2), art. M2. doi: http://doi.org/10.7203/relieve.24.2.13374

$\begin{gathered}\text { Revista ELectrónica de } \\ \text { Investigación y EValuación } \\ \text { Educativa }\end{gathered}$
ISSN: 1134-4032

\title{
La transformación del currículo en una sociedad diversa: ¿quién y cómo se decide el currículum?
} Curriculum transformation in a diverse society: Who decides curriculum, and how?

\author{
Sleeter, Christine \\ California State University Monterey Bay
}

\begin{abstract}
The purpose of this article is to show how, in the United States, some states and school districts are transforming their curriculum through ethnic studies. Ethnic studies exists as a result of unequal power relations in which historically marginalized groups have not had the power to define how education will serve their own communities. According to its activists, ethnic studies not only teaches about the cultures of diverse groups, but also reconstructs, the curriculum around the counter-narratives perspectives, epistemologies, and visions of groups that have been treated historically as if their experiences and perspectives were of lesser value. The article begins with a theoretical framework that considers school knowledge as filtered through the perspectives of dominant groups. Sleeter then reviews research on the perspectives that structure the knowledge in school textbooks, showing how it represents narratives of dominant groups, and the impact of those perspectives on minoritized students. Then Sleeter considers ethnic studies as counter-narrative, and reviews research on the academic impact of ethnic studies on students. A limitation of the implementation of ethnic studies is that it addresses specific courses rather than transforming the whole curriculum. Sleeter developed a framework to help teachers transform their lessons and units in all disciplines, using the central ideas of ethnic studies; this framework is presented. The article concludes with a few implications for Spain from this work in the United States
\end{abstract}

Keywords: curriculum; ethnic studies; counter-narratives; curricular transformation

\begin{abstract}
Resumen
El propósito de este artículo es demonstrar cómo, en los Estados Unidos, algunos estados y distritos escolares están transformando su currículo a través de los estudios étnicos. La existencia de estudios étnicos es el resultado de relaciones de poder desiguales en las cuales los grupos históricamente minorizados no han tenido el poder de definir cómo la educación puede servir a sus propias comunidades. Según sus activistas, los estudios étnicos no solo enseñan sobre las culturas de grupos diversos, pero también reconstruyen el currículo en torno a las contra-narrativas, perspectivas, epistemologías y visiones de grupos tratados históricamente como si sus experiencias y perspectivas fueran de menor valor. El artículo comienza con un marco teórico que considera los conocimientos escolares como filtrados desde las perspectivas de los grupos dominantes. Sleeter resume las investigaciones sobre las perspectivas que estructuran el conocimiento en los textos escolares, que representan narrativas de los grupos dominantes, y el impacto de estas perspectivas en los estudiantes minorizados. Luego Sleeter considera los estudios étnicos como contra-narrativas, y resume las investigaciones en el impacto académico de los estudios étnicos en los estudiantes. Una limitación es que la implementación de los estudios étnicos aborda cursos específicos en lugar de transformar el currículo en total. Sleeter desarrolló un marco para ayudar a los profesores en la transformación de sus lecciones y unidades en todas las disciplinas, utilizando las ideas centrales de los estudios étnicos; este marco está presentado. El artículo concluye destacando algunas implicaciones para España de este trabajo en los EE UU refugiados.
\end{abstract}

Palabras clave: currículo; estudios étnicos; contra-narrativas; transformación curricular

\section{Reception Date}

2018 September 11

Approval Date 2018 December 07

Publication Date:

2018 December 24

Fecha de recepción 2018 Septiembre 11

Fecha de aprobación 2018 Diciembre 07

Fecha de publicación 2018 Diciembre 24 
En 2016, el Estado de California adoptó una ley que autoriza el desarrollo de un currículum en los estudios étnicos para ser usado en todo el Estado. Es decir, para escuelas secundarias se desarrollará un curso, escrito desde las perspectivas de grupos raciales y étnicos marginados. El autor de esta ley, Luis Alejo, fue motivado por su experiencia de transformación personal cuando se especializó en Estudios Chicanos y Ciencias Políticas en la Universidad de California - Berkeley. Aunque esta ley se refiere a solo un curso en el nivel secundario, representa el comienzo de un cambio significativo que tiene lugar en los EE UU con respecto a las perspectivas que se representen en el currículo escolar.

En los EE UU, los estudios étnicos nacieron en la década 1960 en comunidades minoritarias raciales en California. Este movimiento vio el currículo universitario como fuerza colonizadora, e irrelevante para solucionar los problemas comunitarios porque no ofrecía un análisis del racismo y otras inequidades estructurales. Los activistas exigieron un currículo universitario relevante impartido por profesores de comunidades vulneradas. Treinta años más tarde, un grupo de ciudadanos mexicano-americanos solicitó al distrito escolar en Tucson, Arizona un plan de estudios básicos y secundarios que se centrara en los Estudios MexicanoAmericanos. Es decir, en la historia y la literatura escrita por autores e intelectuales con ascendencia mexicana que escriben desde el punto de vista de las comunidades mexicanaamericanas. Estaban hartos que las escuelas siguieran sirviendo mal a sus hijos. En 1998, las escuelas de Tucson lanzaron un programa de Estudios Mexicano-Americanos. Fue el primer programa de estudios étnicos primarios y secundarios de todo un distrito escolar.

La existencia de estudios étnicos es el resultado de relaciones de poder desiguales en las cuales los grupos históricamente minorizados no han tenido el poder de definir cómo la educación puede servir a sus propias comunidades, en un contexto de luchas más amplias por derechos, recursos y oportunidades. Según sus activistas, los estudios étnicos no solo enseñan sobre las culturas de grupos diversos, pero también reconstruyen el currículo en torno a las contranarrativas, perspectivas, epistemologías y visiones de grupos tratados históricamente como si sus experiencias y perspectivas fueran de menor valor.

Este artículo tiene cinco secciones. La primera parte presenta un marco teórico que considera los conocimientos escolares como filtrados desde las perspectivas de los grupos dominantes. La segunda parte resume las investigaciones sobre las perspectivas que estructuran el conocimiento en los textos escolares, y el impacto de estas perspectivas en los estudiantes minorizados. La tercera parte considera los estudios étnicos como contranarrativas. Luego, consideramos un marco para transformar lecciones y unidades en todas las disciplinas, utilizando las ideas centrales delos estudios étnicos. El artículo concluye destacando algunas implicaciones para España de este trabajo en los EE UU.

\section{Marco teórico}

El currículo enseña una manera de mirar el mundo: "las interpretaciones de sentido común que usamos” (Apple, 2004, pág. 5) incluyendo las interpretaciones del sistema social, de personas como uno mismo y de personas que consideramos diferentes. El análisis de Apple (2004) de la relación entre la estructura de una economía capitalista, y la formación de una conciencia que acepta el capitalismo y la propia posición dentro del sistema de clase social, es útil. Su trabajo se centra en el rol mediador del currículo, argumentando que no es neutral ideológicamente. Por el contrario, lo que se enseña en las escuelas representa una selección de una gama más amplia del conocimiento disponible que se podría enseñar. ¿Quién filtra ese conocimiento, y con qué propósito?

Brown y Brown (2015) describen el currículo como "la fabricación de la memoria, o la forma en la que una nación imagina y da forma a lo que las personas llegan a saber sobre el pasado y el presente" (pág. 104). Se preguntan de quién es la memoria escrita en el currículo. 
Gran parte del currículo sirve para el proyecto de construir la nación y de mantener la cohesión social en torno de las relaciones de poder particulares. La enseñanza de un currículo oficial que refleja la ideología de los grupos sociales poderosos contribuye a la hegemonía, o "el conjunto organizado de significados y prácticas" que mantienen el poder y el control (Apple, 2004, pág. 110).

Generalmente, los proyectos de construcción de naciones construyen narrativas basadas en mitos de origen e identidad de los grupos dominantes, y se esfuerzan por construir una identificación psicológica personal con esa narrativa (Van Alphen \& Carretero, 2015). La gente que ocupan posiciones de poder generalmente aceptan como sentido común tales historias de orígenes, identidades, $\mathrm{y}$ cultura.

Pero ¿qué pasa cuando la narrativa que se enseña en la escuela entra en conflicto con las narrativas cotidianas aprendidas en la propia comunidad? ¿La incapacidad de las personas de "ver su propio rostro en el espejo del pasado" de los conocimientos escolares (Carretero, 2011, pág. 36), pone en duda la utilidad o la legitimidad de estos conocimientos? Las contra-narrativas de grupos que ocupan posiciones sociales marginadas desafían cosmovisiones e ideologías dominantes sobre la nación y sus relaciones de poder. Generalmente estas contra-narrativas se construyen y se enseñan en hogares y las comunidades en lugar de en las escuelas. Por ejemplo, Gallegos (1998) describió las enormes diferencias entre la ideología sobre los EE UU en el currículo que experimentó durante su escolarización, y la ideología que aprendió de los miembros de la familia mexicanos e indígenas. "Las historias sobre cómo funciona el mundo que escuché y aprendí mientras crecía son tal radicalmente diferentes de las explanaciones que aprendí en las instituciones, ambas son casi irreconciliables" (pág. 244).

La figura 1 yuxtapone puntos de vista que son común en cómo los miembros de grupos dominantes, y los miembros de grupos subordinados perciben la sociedad. Aunque la perspectiva dominante retrata el orden social como legítimo y a los miembros de grupos dominantes como positivos, no hace los mismo con miembros de grupos subordinados. Sin embargo, para mantener control social, el grupo dominante tiene que persuadir a los miembros de grupos subordinados para que acepten la legitimidad del orden social que existe, incluso cuando eso signifique percibirse a sí mismos como incapaces, atrasados, de menor inteligencia o débiles.

\begin{tabular}{|c|c|c|}
\hline $\begin{array}{c}\text { Naturaleza de } \\
\text { la sociedad }\end{array}$ & La perspectiva dominante & La perspectiva subordinada a todos \\
\hline $\begin{array}{c}\text { Naturaleza de } \\
\text { los grupos } \\
\text { dominantes }\end{array}$ & $\begin{array}{c}\text { Trabajan duro, inteligente, ganaron } \\
\text { su posición, fuerte }\end{array}$ & $\begin{array}{c}\text { Tienen privilegios que no reconocen, } \\
\text { no tratan a los otros justamente, solo } \\
\text { se preocupan por ellos mismos }\end{array}$ \\
\hline $\begin{array}{c}\text { Naturaleza de } \\
\text { los grupos } \\
\text { subordinados }\end{array}$ & $\begin{array}{c}\text { Muchos carecen de valores, respeto, } \\
\text { iniciativa, inteligencia; pueden ser } \\
\text { buenos para el trabajo manual }\end{array}$ & $\begin{array}{c}\text { Fuertes, resilientes e ingeniosos, } \\
\text { tienen una larga historia de } \\
\text { conocimiento comunitario y familiar }\end{array}$ \\
\hline
\end{tabular}

Figura 1 - Contraste de perspectivas

Si una narrativa curricular oficial ignora o deja a un lado las otras narrativas, ¿qué significaría transformar el currículo? Carretero (2011) examinó esta cuestión en relación al currículo de historia, que tradicionalmente ha tenido el propósito de fomentar el patriotismo. Cuando la historia oficial entra en conflicto con las diversas narrativas cotidianas dentro de 
y afuera de las fronteras de una nación, ¿̇quién debe decide el currículum? Carretero sugiere no reemplazar una verdad absoluta con otra, sino buscar "una explicación relacional o contextual de las 'verdades' elaboradas o producidas por la historia” (pág. 201).

La transformación del currículo implica la enseñanza desde diferentes perspectivas y puntos de vista. Según Banks (2004), "El conocimiento que emana de comunidades epistemológicas marginadas a menudo cuestiona las prácticas políticas, económicas y educativas existentes, y exige cambios y reformas fundamentales. A menudo revela la inconsistencia entre los ideales democráticos dentro de una sociedad y sus arreglos sociales y prácticas educativas” (pág. 237).

critica el orden social y la naturaleza de los grupos dominantes, ya que se basa en la sabiduría histórica y las ideas de las comunidades subordinadas. Debido a que este conocimiento cuestiona la legitimidad de relaciones de poder desiguales, normalmente las contra-narrativas, perspectivas, epistemologías y culturas de personas que han sido minorizadas, o las de grupos considerados como "otros," históricamente son tratadas como si sus experiencias y perspectivas fueran de menor valor. El discurso "peligroso" es marginado. Como resultado, Besalú Costa (2016) explica que la mayoría del discurso curricular en España representa las perspectivas orientadas hacia la cohesión en lugar de la crítica social:

Desde finales de la primera década del nuevo siglo casi ha desaparecido del discurso público lo intercultural y su lugar lo han ido ocupando progresivamente términos como cohesión social y convivencia. El multiculturalismo, la interculturalidad, entendidos como el reconocimiento pleno del derecho de las minorías a mantener y desarrollar su propia identidad, a participar en pie de igualdad en los asuntos públicos, a formar parte a todos los efectos del cuerpo social, se considera perjudicial en la práctica, porque - se argumenta - podría erosionar la convivencia y fracturar la cohesión social, porque abriría una puerta que podría propiciar la división social y el enfrentamiento por motivos étnicos, culturales o religiosos (pág. 29).

En los EE UU, como mostraré, aunque la mayoría de los currículos representan las perspectivas de grupos con poder (blanco, afluente), en algunas comunidades las contranarrativas desde los puntos de vista de las comunidades minorizadas transforman el currículo de una manera que produce un impacto muy positivo en los estudiantes.

\section{Análisis del curriculum y su impacto en los estudiantes}

En los EE UU, los textos (y otros documentos curriculares en menor grado) se han analizado durante décadas para determinar qué grupos se incluyen y excluyen, cuáles perspectivas predominan, y cómo se representan los diversos grupos. Un método sencillo de análisis consiste en contar las personas que salen en las imágenes, las personas que se nombran como objeto de estudio, o los personajes principales de cuentos literarios, identificando cada una por raza y sexo (Grant \& Sleeter, 2009, pág. 128-134). Se puede también prestar atención a cómo se representa cada grupo: qué características tienen o qué roles ocupan. Aunque este método de recuento no examina directamente los principios de ordenamiento que estructuran el currículo, sí indican qué perspectiva domina

Los análisis curriculares en los EE UU descubren que las personas blancas continúan recibiendo la mayor atención, apareciendo en una variedad más amplia de roles y dominando las historias y las listas de logros. Si bien el tratamiento de grupos minorizados ha mejorado con el tiempo, la perspectiva curricular sigue siendo blanca. Los afroamericanos aparecen en una gama más limitada de roles que los blancos, y aparecen episódicamente en vez de dentro de una narrativa más amplia de las experiencias afroamericanas (Pelligrino, Mann \& Russell, 2013). Más o menos, solo 3\% de las oraciones de textos de las ciencias sociales se relacionan con latinos (Noboa, 2005), y los textos literarios incluyen autores latinos, pero 
presentan los mismos pocos y aún se basa en estereotipos (Rojas, 2010). Los indígenas siguen estando muy pocos representados, simplificado, ubicados en el pasado, y puestos en un rol pasivo (Stanton, 2014). Asiáticos americanos y árabe americanos solo hacen apariciones limitadas y a menudo estereotipadas (Romanowski, 2009). Los textos dicen muy poco sobre el racismo contemporáneo, generalmente blanqueando en gran medida lo que mencionan.

Un método más complejo de análisis curricular consiste en comparar el tratamiento de las ideas, los eventos, o las personas en los textos con los libros y otros recursos que fueron escritos por miembros de comunidades minorizadas. En su análisis de las narrativas en los textos, Brown y Brown (2010) empezaron con períodos de tiempo clave y narrativas de la historia afroamericana, tal como han sido escritos por intelectuales afroamericanos. Luego, examinaron de cerca los textos de historia de EE UU, observando cómo cada texto trataba esos períodos de tiempo específicos. De esa manera, su análisis comparó las perspectivas desde las cuales los textos fueron escritos con las de los historiadores afroamericanos. Brown y Brown descubrieron que, si bien los textos incluyen información correcta sobre la historia afroamericana en cada uno de los períodos analizados, "estas representaciones no llegan a ilustrar adecuadamente cómo la violencia racial opera sistemáticamente para oprimir y reducir las oportunidades de los afroamericanos y su movilidad en los Estados Unidos” (pág. 150).

En España, encontramos algunos análisis similares. Samper Rasero y Garreta Bochaca (2011) explican que los pocos estudios existentes "generalmente se centran en los contenidos transmitidos, las imágenes que los soportan, y el idioma utilizado” (pág. 83). Estos autores tenían interés en cómo el currículo sitúa a los árabes y el islam en la historia de Andalucía. Explican que Andalucía se puede ver como una parte integral de España a través de del cual los árabes pasan de vez en cuando, o íntimamente entrelazado con el norte de África. Aunque la segunda perspectiva ve una fraternidad hispano-árabe, la primera ve el conflicto y el antagonismo entre dos culturas muy distintas. Los autores analizaron 246 documentos curriculares aprobados, incluidos textos, en Cataluña. Descubrieron que la mitad no tenía alguna referencia al islam, 9.5\% tenía más que un extracto, y solo 1.5 tenía un capitulo. o sección, entero dedicado al islam. Cuando se representaba el islam y/o los árabes, generalmente no se mostraban en Cataluña (y menos aún en España), sino en otro lugar (por ejemplo como parte de la historia mundial). El islam se representaba principalmente a través de fotos o dibujos en lugar de oraciones o párrafos. Las imágenes sugirieron que "el islam es radicalmente exótico. . . incompatible con los valores occidentales" (pág. 88). y también es antimoderno. Este y Oeste se mostraron como culturas separadas, independiente una de otra. Más o menos una mitad de las referencias a los musulmanes en Cataluña se refieren a la inmigración, que se representa como un problema. En todo, su análisis descubrió que los textos enmarcan Andalucía como algo separado y distinto culturalmente del norte de África, y los norteafricanos como extranjeros cuya inmigración a España crea problemas.

Cómo los estudiantes experimentan el currículo depende en parte de las perspectivas que se representan, y en parte en cómo se ven a sí mismos representados. En los EE UU, se han realizado algunas investigaciones sobre cómo los estudiantes de grupos minorizados perciben el currículo. La mayoría de estas investigaciones se ha realizado con estudiantes afroamericanos. Al nivel primario, muchos estudiantes afroamericanos notan discrepancias entre puntos de vista en el currículo y lo que aprenden en el hogar. A los niveles medio y secundaria, muchos consideran que la blancura del currículo contribuye a su desconexión (Epstein, 2009). Igualmente, estudiantes mexicanoamericanos e indígenas generalmente consideran que el currículo tradicional es alienante (Martinez, 2010; Ochoa 2007). 


\section{Los estudios étnicos como contra-narrativas}

En Tucson, Arizona, debido al trabajo de unos ciudadanos mexicano-americanos, entre 1998 y 2012, el Departamento de los Estudios Mexicano-Americanos del distrito escolar trabajó con las escuelas locales para fortalecer el aprendizaje mediante el desarrollo de una amplia gama de recursos curriculares que se alinearon con los estándares de contenido curricular establecidos por el estado, y se centraron en los marcos intelectuales de los estudios mexicano-americanos. El currículo que se desarrollaba desafía la narrativa dominante. Por ejemplo, un programa de secundaria fue el Proyecto de Educación para la Justicia Social. El proyecto se basó en un modelo del "intelectualismo críticamente consciente” para fortalecer el aprendizaje de los estudiantes mexicano-americanos (Cammarota \& Romero, 2009). El modelo incluye: 1) un currículo académico riguroso que se alinea con los estándares, es relevante cultural e históricamente para los estudiantes, y se enfoca en la justicia social; 2) la pedagogía crítica en la que los estudiantes desarrollan el pensamiento crítico y la conciencia crítica (siguiendo a Paulo Freire), creando los conocimientos más que consumiéndolos; y 3) el cuidado auténtico a través del cual los docentes demuestran un profundo respeto por los estudiantes $\mathrm{y}$ sus familias como intelectuales. El currículo sumergió a los estudiantes en lecturas teóricas al nivel universitario, e incluyó un proyecto de investigación participativa en la comunidad. Los estudiantes recopilaron datos sobre las manifestaciones del racismo en su escuela y su comunidad, utilizando teoría de las ciencias sociales para analizar patrones en sus datos, y proponer soluciones a los problemas. Ellos presentaban ponencias formales de los resultados de sus investigaciones a miembros de la comunidad, en conferencias de jóvenes, $\mathrm{y}$ en congresos

El impacto del programa en los estudiantes fue examinado minuciosamente, inicialmente para documentar su éxito en la mejora de los logros de los estudiantes, y más tarde para defender su existencia. Cabrera y sus colegas
(2014) compararon los logros y las tasas de graduación de los estudiantes de grados $11 \mathrm{y}$ 12 que experimentaron y no experimentaron cursos de Estudios Mexicano-Americanos. Según sus resultados, aun cuando los estudiantes que participaron en el programa entraron con calificaciones más bajas en comparación a los estudiantes que no participaron, en el grado 12 alcanzaron tasas significativamente más altas de aprobación en las pruebas estandarizadas del estado y de graduación.

Sin embargo, debido a su temor que el programa enseñara sedición, unos líderes políticos blancos al nivel del estado lograron la aprobación de una ley que prohibía los estudios étnicos en el estado; así 5 años más tarde esta ley fue anulada. Pero la lucha por los estudios étnicos en Tucson lanzó un movimiento nacional de base popular para la transformación de la educación a través de los estudios étnicos. En 2014, un distrito escolar en el sur de California votó por adoptar estudios étnicos como requisito de graduación de la escuela secundaria; este movimiento rápidamente se extendió por California. Hoy, existen esfuerzos similares en otros estados tales como Washington, Rhode Island, Nuevo México y Kansas.

Con este interés nacional, mis colegas y yo nos preocupamos que las escuelas van a adoptar o crear versiones de los estudios étnicos que reflejan perspectivas dominantes. Por ejemplo, hay un plan de estudios en California que conceptualiza los estudios étnicos como la enseñanza de un grupo étnico a la vez, cada grupo recibe más o menos cuatro semanas de atención. Pero esta versión no sitúa a los grupos dentro de un análisis de las estructuras del racismo; no aborda las relaciones entre los profesores y los estudiantes de grupos minoritarios; no aborda las expectativas académicas para su logro académico; y no desarrolla una crítica de los temas centrales del currículo en su conjunto.

Hemos elaborado un marco conceptual que será publicado en un libro que estamos en proceso de coordinación, Rethinking Ethnic Studies (Zavala, Cuauhtin, Sleeter \& Au). El 
fulcro de este marco es una doble hélice con dos partes: la recuperación de la humanidad (de los estudiantes, sus familias, sus comunidades), a través de la criticidad (en el sentido de Freire). Hemos identificado 4 conceptos básicos que se reflejan en la literatura académica de los estudios étnicos y también en el trabajo de los activistas. Proponemos que estos conceptos deben ser la fundación de un currículo de los estudios étnicos:

1. La Indigenidad / las raíces. La soberanía de los pueblos indígenas en los EEUU y en todo nuestro planeta, que aún mantienen relaciones con sus bases en tierras ancestrales y culturas ancestrales, es una consideración primordial. Cada ser humano tiene raíces indígenas ancestrales en los diversos continentes de nuestro planeta. En otras palabras, los estudiantes (y los profesores) necesitan reconocer quien tiene la indigenidad en este lugar, y de dónde son nuestras raíces indígenas.

2. Crítica de la colonización / la deshumanización. Mientras los pueblos se han trasladado a lo largo de la historia humana y el conflicto y la conquista siempre han ocurrido, a partir de 1492 el mundo occidental tomó la conquista a un nivel global. En el proceso creó nuevas construcciones sociales de raza y jerarquía racial, que continúan teniendo reverberaciones. Es importante estudiar las raíces de este sistema, y sus manifestaciones actuales para reconocer los procesos que deshumanizan a las comunidades y a los estudiantes en el sistema escolar; procesos que pueden ser cambiados.

3. La hegemonía y las contra-narrativas. Los estudios étnicos ofrecen historias de oposición y contra-narrativas, nombrando, hablando y resistiendo el racismo, la colonización, la pobreza, y los sistemas entrelazados de opresión. Los estudiantes aprenden a identificar y a criticar las narrativas dominantes en relación a sus vidas, $\mathrm{y}$ aprenden las narrativas alternativas y los conocimientos ancestrales de sus comunidades.

4. La regeneración, la transformación. Considerando que el propósito de los estudios étnicos es eliminar el racismo y todas las formas entrelazadas de opresión, los estudios étnicos orientan hacia la descolonización de la mente, una revitalización, celebración y recuerdo de las raíces indígenas y un análisis crítico de la opresión. El currículo regenerativo ayuda a los estudiantes a superar los impactos tóxicos de la amnesia histórica y los procesos de colonización y control social sobre ellos mismos, y reflexionar sobre sus identidades y futuros. Los jóvenes son tratados intencionalmente como intelectuales que vienen a constituirse como agentes activos en sus propias comunidades, utilizando el conocimiento como herramienta en su trabajo por la justicia social y la liberación.

Cuando estos conceptos constituyen una parte sustantiva del currículo, los estudiantes se benefician. Las investigaciones documentan que, para los estudiantes afroamericanos, mexicano-americanos, e indígenas, los estudios étnicos producen un impacto positivo en su participación en el aula, sus logros en las pruebas y su sentido de empoderamiento. En 2010, la Asociación Nacional de la Educación - el sindicato de profesores en los EEUU - me encargó preparar un informe sobre las investigaciones del impacto de los estudios étnicos en los estudiantes. El sindicato quería esta información para defender a los profesores en Tucson, donde el gobierno estaba en el proceso de terminar el programa en los Estudios Mexicano-Americanos. Desde que escribí mi informe (Sleeter, 2011), se han publicado otras dos investigaciones importantes (Cabrera et al., 2015; Dee \& Penner, 2017).

En síntesis, tres investigaciones documentaron altos niveles de participación en el aula cuando el docente utilizó literatura escrita por los autores de la etnia de los estudiantes. Las investigaciones sobre cinco programas de alfabetización (3 para 
estudiantes afroamericanos de secundaria y 2 para estudiantes indígenas de primaria) documentaron un crecimiento de las habilidades de alfabetización. Las investigaciones sobre dos programas para las matemáticas/las ciencias (para estudiantes indígenas) encontraron un impacto positivo en el rendimiento académico y en las actitudes hacía el aprendizaje. Las investigaciones sobre seis currículos (4 en las ciencias sociales, 1 en literatura, y 1 en "habilidades para la vida") encontraron un impacto positivo en el rendimiento académico y en el sentido de agencia de los estudiantes. Sólo una investigación no encontró un impacto positivo, principalmente debido a un conflicto entre cómo el currículo conceptualizó la cultura afroamericana, y cómo los estudiantes la experimentaban diariamente.

Los programas de estudios étnicos diseñados para estudiantes diversos que incluían a estudiantes blancos, tenían un enfoque primario en influenciar la comprensión de los estudiantes acerca del racismo o su conocimiento de otros grupos. Según las investigaciones, la simple de adición de representaciones de diversas personas en el currículo afecta sólo marginalmente a las actitudes de los estudiantes, porque las actitudes se adquieren de manera activa en lugar de pasiva. Lecciones que directamente enseñan sobre el racismo producen un impacto más fuerte que lecciones que incluyen grupos diversos pero ignoran el racismo. Un gran cuerpo de las investigaciones universitarias reporta bastante consistentemente que estos cursos afectan positivamente a los estudiantes cuando incluyen la interacción entre grupos.

Debido al éxito documentado de estos programas, en este momento hay un movimiento nacional en comunidades minoritarios para adoptar cursos y requisitos de estudios étnicos. Sin embargo, la adición de uno o dos cursos que representan las perspectivas de comunidades subordinadas no constituye la transformación de todo del currículo, y no aborda necesariamente el problema de reemplazar una narrativa por otra.

\section{Hacia un proceso de transformar el curriculum}

En mi trabajo con profesores, desarrollé un marco y un proceso para ayudar a los profesores a transformar su currículo (Sleeter \& Flores Carmona, 2016). El proceso que describiré implica la transformación de una unidad curricular que un profesor planea enseñar. El proceso ayuda a los profesores a conectar su currículo con su contexto local; e implica considerar cómo trabajar con los conocimientos de los estudios étnicos. El marco se ilustra en la figura 2

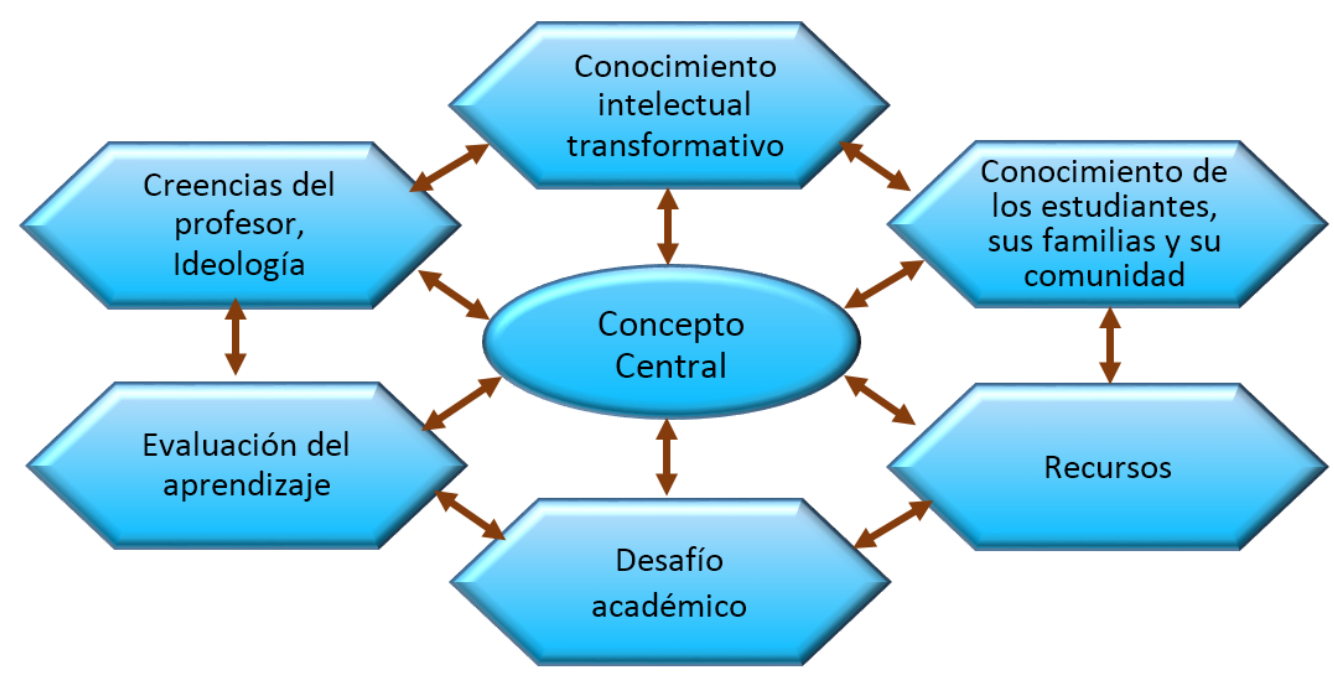

Figura 2. Marco para el diseño del currículo multicultural 
Los profesores empiezan con la identificación de “comprensiones duraderas” que planean enseñar, utilizando el concepto de “diseño hacía atrás” (Wiggins \& McTighe 2005). Wiggins y McTighe (2005) definen las comprensiones duraderas como "las grandes ideas que tienen un valor duradero más allá del aula (...) residen en el centro de la disciplina, y son transferibles a situaciones nuevas” (pág. 342). Los profesores pueden identificar las comprensiones duraderas, o conceptos centrales, en cualquier disciplina. Estas comprensiones forman la base de la planificación curricular. El diseño hacía atrás se refiere a un proceso de planificación que empieza con la identificación de las comprensiones duraderas (conceptos centrales), y cómo se ve cuando los estudiantes demuestran su comprensión. El diseño de la evaluación del aprendizaje debe partir de este análisis, de cómo se ve el aprendizaje cuando lo demuestran. Luego, el profesor considera qué tipo de currículo y experiencias permitirán a los estudiantes adquirir cada comprensión duradera y demostrar sus nuevos conocimientos.

Este comienzo útil obliga a los profesores a analizar su currículo - sus estándares, sus libros de texto, el alcance y la secuencia curricular - para descubrir exactamente qué es lo que pretenden que sus estudiantes ganan de la enseñanza. En el proceso de análisis curricular, generalmente los profesores descubren una flexibilidad que no sabían que tenían. Con la vista puesta en el aprendizaje de los estudiantes en lugar de la cobertura de contenidos, podemos preguntar ahora qué contenidos y experiencias involucrarán mejor a sus estudiantes en el aprendizaje.

El conocimiento intelectual transformativo se refiere a los "conceptos, paradigmas, temas y explicaciones” que surgieron en las florecientes tradiciones críticas de las escrituras en los estudios étnicos y los estudios de las mujeres (Banks, 1993, pág. 9). El marco conceptual para los estudios étnicos que se describió anteriormente sintetiza cuatro conceptos fundamentales que residen dentro del conocimiento intelectual transformativo. Si bien es útil que los profesores conozcan estos conceptos, les resulta más útil estar atentos a los temas en las obras que leen. Les pido a los profesores que investiguen sobre el conocimiento de los estudios étnicos que se relaciona directamente con el concepto central de las lecciones o la unidad que están planeando. Tienen que leer literatura académica producida por un grupo marginalizado históricamente en relación con ese concepto; por lo general, necesitan ayuda en identificar qué leer. Escriben un breve ensayo que no solo describe los contenidos que pueden incluir, sino que también examina la perspectiva en la literatura sobre el concepto central en sí mismo.

Les pido a los profesores que investiguen los conocimientos que sus estudiantes traen a la escuela desde su hogar y su comunidad, y organicen sus lecciones de tal manera que los estudiantes pueden activar y utilizar ese conocimiento. Por ejemplo, profesores pueden entrevistar a unos estudiantes, preguntando qué ya saben, o creen que saben, sobre la idea central que el profesor planea enseñar. Por lo general, tales entrevistas revelan una combinación de suposiciones incorrectas, preguntas inesperadas que a los estudiantes les gustaría explorar, y conocimiento previo que los estudiantes ya tienen sobre el cual el profesor puede construir.

El rigor intelectual se refiere a la medida en la que la unidad desafía a los estudiantes intelectualmente. Un gran problema son las bajas expectativas de los estudiantes minorizados y los de comunidades vulneradas. Por eso, involucro a los profesores en un examen crítico de sus expectativas académicas de sus estudiantes; utilizo la taxonomía de Bloom como una herramienta para analizar su propio currículo. Consideramos cómo sería su enseñanza si los profesores prepararan a sus estudiantes para la universidad, y los apoyan en su aprendizaje. Los profesores desarrollan estrategias de andamiaje para usar con sus estudiantes mientras aprenden a hacer un trabajo académico complejo.

Este enfoque para transformar el currículo tiene una gran limitación en comparación con 
la creación y enseñanza de cursos en los estudios étnicos: el conocimiento del profesor en sobre el cuerpo de textos académicos escrito por miembros de los grupos minorizados. La investigación anterior en el conocimiento de los estudios étnicos que está directamente relacionada con el concepto central que el profesor planea enseñar, representa solo un comienzo de un proyecto más amplio en el aprendizaje del punto de vista, experiencias, conocimiento ancestral, y conocimiento intelectual de un grupo cuyas perspectivas han sido subyugadas. En California, algunas universidades están experimentando con el requisito que los profesores en formación realicen un curso de estudios étnicos (por ejemplo, la historia afroamericana) para desarrollar su base conocimientos. También, algunas universidades en los EE UU tienen programas para preparar más profesores de las comunidades minorizadas.

\section{Implicaciones para España}

En los EE UU, los estudios étnicos abordan relaciones de poder desiguales que se basan en la raza y la etnia al transformar el currículo en torno a las perspectivas y los conocimientos de grupos minorizados, y al enseñar a los estudiantes de estos grupos como intelectuales y futuros ciudadanos activos. Investigaciones han descubierto consistentemente que este tipo de transformación tiene un impacto positivo en los estudiantes. Si bien la mayoría del trabajo en los estudios étnicos en las escuelas secundarias implica la creación de un curso nuevo (generalmente en las ciencias sociales), lo que se necesita es una transformación integral del currículo, la pedagogía, y las relaciones entre los profesores y las comunidades que sirven.

Obviamente, hay muchas diferencias entre España y los EE UU. No sugiero que se puede transportar las prácticas de un contexto a otro. Sin embargo, creo que hay ideas de lo que sucede en los EE UU que podría ser útil para los educadores en España.

España tiene una población diversa. En 2011 más o menos $12 \%$ de la población española eran inmigrantes, la mayor cantidad provenía de Europa del Este, Marruecos, y
América Latina. Además, hay diversidad de género, ascendencia, región, y clase social. De igual importancia a la diversidad cultural y lingüística es la consideración de las relaciones de poder, especialmente el poder de definir cómo se representan a las familias y las comunidades, y los derechos y oportunidades que tienen

Reflexionando sobre el análisis de los libros de texto discutido anteriormente, la pregunta principal fue: ¿desde qué punto de vista se les enseña a los estudiantes a ver el mundo? $\dot{A}$ A verse a sí mismos? La figura 1 plantea la consideración que los textos retratan la sociedad como justa para todos, y las comunidades subordinadas en términos de características personales, culturales e intelectuales que les faltan. Si esto es cierto, tenemos que preguntar, ¿qué tipo del impacto tiene esta perspectiva en los estudiantes minorizados? ¿En los estudiantes españoles de grupos dominantes?

En sus investigaciones, Aguado, Ballestreros y Malik (2003) descubrieron que, "En general, el conocimiento y las actitudes de los estudiantes hacia la diversidad cultural es muy estereotipado, y se limita a los grupos presentes en sus clases” (pág. 57). Para los estudiantes de los grupos dominantes, sus perspectivas sobre el orden social, su desigualdad, y "otros" grupos reflejan estereotipos. Los estudiantes de los grupos subordinados experimentan problemas, pero no tienen un marco crítico para analizarlos. Por ejemplo, Aguado, Ballestreros, y Malik escriben que "Algunos niños que no van bien en la escuela, afirman que . . .'Trabajo poco porque no me interesa lo que dicen, creo que son cosas inútiles”" (pág. 57). Además, las autoras notan que, "En general, los profesores perciben las diferencias culturales como un problema y una amenaza. Ellos intentan incluso evitar hablar de las diferencias, por miedo a promoverlas” (pág. 58).

Basados en nuestra experiencia con los estudios étnicos en los EE UU, sugiero que existen perspectivas subalternas que, cuando forman parte del currículo escolar, pueden replantear cómo los estudiantes de los grupos 
subordinados se ven a sí mismos y su futuro. Si bien aprender a ver el mundo a través de los ojos de los grupos que han sido marginados se puede sentir como una amenaza para los estudiantes de los grupos dominantes, en última instancia, tal aprendizaje conduce al diálogo en un nivel más profundo que el que existe de otra manera.

\section{Referencias}

Aguado, T., Ballestreros, B., \& Malik, B. (2003). Cultural diversity and school equity. A model to evaluate and develop educational practices in multicultural education contexts. Excellence \& Equity in Education 36(1), 5063. doi: https://doi.org/10.1080/10665680303500

Apple, M. W. (2004). Ideology and curriculum, $3^{\text {rd }}$ ed. New York: Routledge Falmer. https://doi.org/10.4324/9780203487563

Banks, J. A. (1993). The canon debate, knowledge construction, and multicultural education. Educational Researcher 22(5), 414. doi: https://doi.org/10.3102/0013189X022005004

Banks, J. A. (2004). Race, knowledge construction, and education in the United States. En J. A. Banks \& C. A. M. Banks (Eds.), Handbook of research on multicultural education (2nd ed., pp. 228239). San Francisco, CA: Jossey Bass.

Besalú Costa, X. (2016). Interculturalidad y convivencia: discursos que anticipan y legitiman prácticas. Una Mirada Intercultural y Para la Equidad y la Justicia Social, 14, 27-33.

Brown, A. L. \& Brown, K. D. (2010). Silenced memories: An examination of the sociocultural knowledge on race and racial violence in official school curriculum. Equity \& Excellence in Education 43(2), 139-154. doi:

https://doi.org/10.1080/10665681003719590

Brown, A. L. \& Brown, K. D. (2015). The more things change, the more they stay the same: Excavating race and the enduring racisms in U.S. curriculum. Teachers College Record 17(14), 103-130.
Cabrera, N. L., Milam, J. F., Jaquette, O., \& Marx, R. W. (2014). Missing the (student achievement) forest for all the (political) trees. American Educational Research Journal 51(6): 1084-1118. doi: https://doi.org/10.3102/0002831214553705

Cammarota, J., \& Romero, A. (2009). The Social Justice Education Project: A critically compassionate intellectualism for Chicana/o students. En W. Ayers, T. Quinn \& D. Stovall (Eds.), Handbook for social justice education (p. 465-476). New York: Routledge.

Carretero, M. (2011). Constructing patriotism. Charlotte, North Carolina: Information Age Publishing.

Dee, T., \& Penner, E. (2017). The causal effects of cultural relevance: Evidence from an ethnic studies curriculum. American Educational Research Journal 54(1): 127$166 . \quad$ doi: https://doi.org/10.3102/0002831216677002

Epstein, T. (2009). Interpreting national history. New York: Routledge.

Gallegos, B. P. (1998). Remembering the Alamo: Imperialism, memory, and postcolonial educational studies. Educational Studies, 29(3), 232-247. doi: https://doi.org/10.1207/s15326993es2903_1

Grant, C. A. \& Sleeter, C. E. (2009). Turning on learning, 5th ed. New York: Wiley.

Martinez, G. (2010). Native pride. Cresskill, NJ: Hampton Press, Inc.

Noboa, J. (2005). Leaving Latinos out of history: Teaching US history in Texas. New York: Routledge.

Ochoa, G. L. (2007). Learning from Latino teachers. San Francisco: Jossey Bass.

Pelligrino, A., Mann, L. \& Russell, W. B., III. (2013). Lift as we climb: A textbook analysis of the segregated school experience. The High School Journal 96: 209-231. doi: https://doi.org/10.1353/hsj.2013.0009

Rojas, M. A. (2010). (Re)visioning U.S. Latino literatures in high school English classrooms. English Education 42(3): 263-277. 
Romanowski, M. H. (2009). Excluding ethical issues from U.S. history textbooks. American Secondary Education 37 (2), 26-48.

Samper Rosero L. \& Garreta Bochaca, J. (2011). Muslims in Catalonian textbooks. Journal of Educational Media, Memory, and Society 3(1), 81-96.

Sleeter, C. E. (2011). The academic and social value of ethnic studies. Washington, DC: National Education Association.

Sleeter, C. E. \& Flores Camona, J. (2016). Unstandardizing curriculum: Multicultural teaching in the standards-based classroom, 2nd ed. New York: Teachers College Press.

Stanton, C. R. (2014). The curricular Indian agent: Discursive colonization and
Indigenous (dys)angency in U. S. history textbooks. Curriculum Inquiry 44(5): 649676. doi: https://doi.org/10.1111/curi.12064

Van Alphen, F. \& Carretero, M. (2015). The construction of the relation between national past and present in the appropriation of historical master narratives. Integrative Psychology and Behavioral Sciences 49, 512-530. doi: https://doi.org/10.1007/s12124-015-9302-X

Wiggins, G., \& McTighe, J. (2005). Understanding by design, 2nd ed. New York: Pearson.

Zavala, M., Cuauhtin, T., Sleeter C., \& Au, W. (Coord.) (Manuscrito en preparación). Rethinking ethnic studies. Milwaukee, WI: Rethinking Schools Ltd.

\begin{tabular}{lr}
\hline \multicolumn{1}{c}{ Authors / Autores } & \multicolumn{1}{c}{$\begin{array}{c}\text { To know more } \\
\text { / Saber más }\end{array}$} \\
\hline Sleeter, Christine (csleeter@gmail.com) & ORCiD \\
Profesora Emérita en el College of Professional Studies de la California State & 0000-0002-4566-8149 \\
University Monterey Bay. Ha sido Presidenta de la National Association for & \\
Multicultural Education y Vice Presidenta de la Division K de la American & \\
Educational Research Association (AERA). Su investigación se h venido & Google \\
centrando en educación multicultural anti-racista, estudios étnicos y en \\
formación del profesorado. Su dirección postal es: 118 1/2 Dunecrest Ave., \\
Monterey CA 93940, USA.
\end{tabular}

\section{RELIEVE}

Revista ELectrónica de Investigación y $\mathbf{E V}$ aluación Educativa E-Journal of Educational Research, Assessment and Evaluation

[ISSN: 1134-4032]

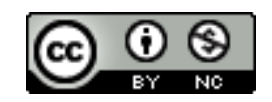

Esta obra tiene licencia de Creative Commons Reconocimiento-NoComercial 4.0 Internacional. This work is under a Creative Commons Attribution 4.0 International license. 